January 22, 2006

\title{
SYMMETRIC PAIRS AND ASSOCIATED COMMUTING VARIETIES
}

\author{
DMITRI PANYUSHEV AND OKSANA YAKIMOVA
}

\section{INTRODUCTION}

The ground field $\mathbb{k}$ is algebraically closed and of characteristic zero. Let $\mathfrak{g}$ be a reductive algebraic Lie algebra over $\mathbb{k}$ and $\sigma$ an involutory automorphism of $\mathfrak{g}$. Then $\mathfrak{g}=\mathfrak{g}_{0} \oplus \mathfrak{g}_{1}$ is the direct sum of $\sigma$-eigenspaces. Here $\mathfrak{g}_{0}$ is a reductive subalgebra and $\mathfrak{g}_{1}$ is a $\mathfrak{g}_{0}$-module. We also say that $\left(\mathfrak{g}, \mathfrak{g}_{0}\right)$ is a symmetric pair. Let $G$ be the adjoint group of $\mathfrak{g}$ and $G_{0}$ the connected subgroup of $G$ with Lie $G_{0}=\mathfrak{g}_{0}$. The commuting variety associated with the involution $\sigma$ or symmetric pair $\left(\mathfrak{g}, \mathfrak{g}_{0}\right)$ is:

$$
\mathfrak{C}\left(\mathfrak{g}_{1}\right)=\left\{(x, y) \in \mathfrak{g}_{1} \times \mathfrak{g}_{1} \mid[x, y]=0\right\} .
$$

The "usual" commuting variety is obtained as a special case of this construction. Indeed, let $(\mathfrak{g} \oplus \mathfrak{g}, \mathfrak{g})$ be the symmetric pair such that $\mathfrak{g}$ is embedded diagonally into $\tilde{\mathfrak{g}}=\mathfrak{g} \oplus \mathfrak{g}$. Then $\tilde{\mathfrak{g}}_{1} \simeq \mathfrak{g}$ as $\mathfrak{g}$-module and $\mathfrak{C}\left(\tilde{\mathfrak{g}}_{1}\right)$ is isomorphic to the commuting variety $\mathfrak{C}(\mathfrak{g})=\{(x, y) \in$ $\mathfrak{g} \times \mathfrak{g} \mid[x, y]=0\}$. The latter was considered by Richardson in [13]. He proved that $\mathfrak{C}(\mathfrak{g})=\overline{G(\mathfrak{t} \times \mathfrak{t})}$, where $\mathfrak{t} \subset \mathfrak{g}$ is a Cartan subalgebra, and therefore $\mathfrak{C}(\mathfrak{g})$ is irreducible. It is not yet known whether $\mathfrak{C}(\mathfrak{g})$ is normal or whether the ideal of $\mathfrak{C}(\mathfrak{g})$ in $\mathbb{k}[\mathfrak{g} \times \mathfrak{g}]$ is generated by quadrics.

Let $\mathfrak{c} \subset \mathfrak{g}_{1}$ be a maximal abelian subspace consisting of semisimple elements. Any such subspace is called a Cartan subspace of $\mathfrak{g}_{1}$. All Cartan subspaces are $G_{0}$-conjugate, see [6]. The rank of the symmetric pair $\left(\mathfrak{g}, \mathfrak{g}_{0}\right)$ is $\operatorname{dim} \mathfrak{c}$, also denoted $\mathrm{rk}\left(\mathfrak{g}, \mathfrak{g}_{0}\right)$. It is known that $\operatorname{dim} \mathfrak{C}\left(\mathfrak{g}_{1}\right)=\operatorname{dim} \mathfrak{g}_{1}+\operatorname{dim} \mathfrak{c}$ and $\mathfrak{C}_{0}:=\overline{G_{0}(\mathfrak{c} \times \mathfrak{c})}$ is an irreducible component of $\mathfrak{C}\left(\mathfrak{g}_{1}\right)$ of maximal dimension, see [10, Sect.3]. It follows that $\mathfrak{C}\left(\mathfrak{g}_{1}\right)$ is irreducible if and only if $\mathfrak{C}\left(\mathfrak{g}_{1}\right)=\mathfrak{C}_{0}$.

The irreducibility problem for $\mathfrak{C}\left(\mathfrak{g}_{1}\right)$ was first considered by Panyushev [10]. He noticed that $\mathfrak{C}\left(\mathfrak{g}_{1}\right)$ can be reducible [10, 3.5]. On the other hand, in some particular cases $\mathfrak{C}\left(\mathfrak{g}_{1}\right)$ is irreducible and enjoys a number of good properties. If $\left(\mathfrak{g}, \mathfrak{g}_{0}\right)$ is a symmetric pair of maximal rank (i.e., $\left.\mathrm{rk}\left(\mathfrak{g}, \mathfrak{g}_{0}\right)=\mathrm{rk} \mathfrak{g}\right)$, then $\mathfrak{C}\left(\mathfrak{g}_{1}\right)$ is an irreducible normal complete intersection and the ideal of $\mathfrak{C}\left(\mathfrak{g}_{1}\right)$ in $\mathbb{k}\left[\mathfrak{g}_{1} \times \mathfrak{g}_{1}\right]$ is generated by quadrics, see [10, 3.2]. We refer to [12], [14], [15] for some recent results.

The problem of irreducibility is closely related to some properties of rings of differential operators. Let $\mathcal{D}\left(\mathfrak{g}_{1}\right)$ denote the algebra of differential operators on $\mathfrak{g}_{1}$ with coefficients in

This research was supported in part by CRDF Grant RM1-2543-MO-03 and RFBI Grant 05-01-00988. 
$\mathbb{k}\left[\mathfrak{g}_{1}\right]$ and $\mathcal{K} \triangleleft \mathcal{D}\left(\mathfrak{g}_{1}\right)$ the left ideal annihilating all $G_{0}$-invariant polynomials. The $G_{0}$-action on $\mathfrak{g}_{1}$ allows us to interpret the elements of $\mathfrak{g}_{0}$ as left-invariant vector fields on $\mathfrak{g}_{1}$. This gives rise to a homomorphism of the universal enveloping algebra $\mathbf{U}\left(\mathfrak{g}_{0}\right)$ to $\mathcal{D}\left(\mathfrak{g}_{1}\right)$. If $\mathfrak{g}_{0}$ contains no simple ideals of $\mathfrak{g}$, then this homomorphism is injective. We may therefore assume that $\mathbf{U}\left(\mathfrak{g}_{0}\right) \subset \mathcal{D}\left(\mathfrak{g}_{1}\right)$. It is easily seen that $\mathbf{U}\left(\mathfrak{g}_{0}\right) \subset \mathcal{K}$. Furthermore, it can be shown that if $\mathfrak{C}\left(\mathfrak{g}_{1}\right)$ is irreducible and its ideal is generated by quadrics, then $\mathcal{K}=\mathcal{D}\left(\mathfrak{g}_{1}\right) \mathbf{U}\left(\mathfrak{g}_{0}\right)$. (See [7] for related results.)

Suppose $\mathfrak{g}$ is simple. Then the known results are

- if $\operatorname{rk}\left(\mathfrak{g}, \mathfrak{g}_{0}\right)=\mathrm{rk} \mathfrak{g}$ (the maximal rank case), then $\mathfrak{C}\left(\mathfrak{g}_{1}\right)$ is irreducible [10];

- if $\operatorname{rk}\left(\mathfrak{g}, \mathfrak{g}_{0}\right)=1$, then $\mathfrak{C}\left(\mathfrak{g}_{1}\right)$ is reducible unless $\left(\mathfrak{g}, \mathfrak{g}_{0}\right)=\left(\mathfrak{s o}_{m+1}, \mathfrak{s o} \mathfrak{o}_{m}\right)$ [14]; furthermore, the number of irreducible components of $\mathfrak{C}\left(\mathfrak{g}_{1}\right)$ equals the number of nonzero nilpotent $G_{0}$-orbits in $\mathfrak{g}_{1}$ [12];

- for $\left(\mathfrak{s l}_{2 n}, \mathfrak{s p}_{2 n}\right)$ and $\left(\mathcal{E}_{6}, \mathcal{F}_{4}\right)$, the commuting variety $\mathfrak{C}\left(\mathfrak{g}_{1}\right)$ is irreducible [12];

- if $\left(\mathfrak{g}, \mathfrak{g}_{0}\right)=\left(\mathfrak{s o}_{m+2}, \mathfrak{s o}_{2} \oplus \mathfrak{s o} \mathbf{o}_{m}\right)$, then $\mathfrak{C}\left(\mathfrak{g}_{1}\right)$ is irreducible [15].

The goal of this article is to present some further results on the irreducibility problem. Let $\mathfrak{g}_{x}$ denote the centraliser of $x \in \mathfrak{g}$. If $x \in \mathfrak{g}_{1}$, then $\sigma$ induces the symmetric decomposition $\mathfrak{g}_{x}=\mathfrak{g}_{0, x} \oplus \mathfrak{g}_{1, x}$, where $\mathfrak{g}_{0, x}:=\mathfrak{g}_{0} \cap \mathfrak{g}_{x}$ is the centraliser of $x$ in $\mathfrak{g}_{0}$. If $h \in \mathfrak{g}_{1}$ is semisimple, then $\left(\mathfrak{g}_{h}, \mathfrak{g}_{0, h}\right)$ is a symmetric pair, which is called a sub-symmetric pair associated with $\left(\mathfrak{g}, \mathfrak{g}_{0}\right)$. Let $\mathfrak{C}\left(\mathfrak{g}_{1, h}\right)$ denote the commuting variety associated with $\left(\mathfrak{g}_{h}, \mathfrak{g}_{0, h}\right)$. Usually, the irreducibility of $\mathfrak{C}\left(\mathfrak{g}_{1}\right)$ is proved in the following way, which goes back essentially to Richardson [13]. First one has to show that $\mathfrak{C}\left(\mathfrak{g}_{1, h}\right)$ is irreducible for any non-zero semisimple $h \in \mathfrak{g}_{1}$; next, one has to verify that $\left(\{e\} \times \mathfrak{g}_{1, e}\right) \subset \mathfrak{C}_{0}$ for any nilpotent element $e \in \mathfrak{g}_{1}$. Actually, the verification in case of nilpotent elements readily reduces to $\sigma$-distinguished elements (see Section 1 for precise definitions). To a great extent, the structure of $\mathfrak{C}\left(\mathfrak{g}_{1}\right)$ depends on properties of $\sigma$-distinguished elements.

In Section 1, we collect several useful facts concerning semisimple and nilpotent elements in $\mathfrak{g}_{1}$. To implement the above program, we should be able to deal with the respective sub-symmetric pairs, and Proposition 1.5 provides a description of all possible sub-symmetric pairs $\left(\mathfrak{g}_{h}, \mathfrak{g}_{0, h}\right)$ in terms of the Satake diagram of $\left(\mathfrak{g}, \mathfrak{g}_{0}\right)$.

In Section 2, we prove the irreducibility of the commuting variety for $\left(\mathfrak{s o}_{n+m}, \mathfrak{s o}_{n} \oplus \mathfrak{s 0 _ { m }}\right)$. This extends the result of [15], which refers to the case $n=2$. The scheme of the proof is similar to that of [15]. But as it often happens, the argument in a general situation is shorter and simpler than in a particular case. Roughly speaking, the reason of success is that, for this symmetric pair, all $\sigma$-distinguished elements are even.

In Section 3, $\mathfrak{C}\left(\mathfrak{g}_{1}\right)$ is shown to be irreducible for the symmetric pairs $\left(\mathfrak{s l}_{2 n}, \mathfrak{s l}_{n} \oplus \mathfrak{s l}_{n} \oplus \mathfrak{t}_{1}\right)$ and $\left(\mathcal{E}_{6}, \mathfrak{s l}_{6} \oplus \mathfrak{s} \mathfrak{l}_{2}\right)$. Here we use the fact that in these two cases $\operatorname{dim} \mathfrak{g}_{1}>\operatorname{dim} \mathfrak{g}_{0}$ and the (closures of) subsets $G_{0}\left(\{e\} \times \mathfrak{g}_{1, e}\right)$ cannot form an irreducible component of $\mathfrak{C}\left(\mathfrak{g}_{1}\right)$. In the 
classical case, $\mathfrak{C}\left(\mathfrak{g}_{1, h}\right)$ is shown to be irreducible by induction, and in the $\mathcal{E}_{6}$-case we rely on the explicit description of all sub-symmetric pairs $\left(\mathfrak{g}_{h}, \mathfrak{g}_{0, h}\right)$.

In [12], it was conjectured that $\mathfrak{C}\left(\mathfrak{g}_{1}\right)$ is irreducible if $\operatorname{rk}\left(\mathfrak{g}, \mathfrak{g}_{0}\right)>1$. This is, however, false. In Section 4, we prove that $\mathfrak{C}\left(\mathfrak{g}_{1}\right)$ is reducible for the symmetric pairs $\left(\mathfrak{s l}_{n+m}, \mathfrak{s l}_{n} \oplus \mathfrak{s l}_{m} \oplus \mathfrak{t}_{1}\right)$ with $n \neq m,\left(\mathfrak{s o}_{2 n}, \mathfrak{g l}_{n}\right)$ with odd $n$, and $\left(\mathcal{E}_{6}, \mathfrak{s o}_{10} \oplus \mathfrak{t}_{1}\right)$. Their ranks are equal to $\min (n, m),[n / 2]$, and 2 , respectively. Furthermore, we give am explicit lower bound on the number of the irreducible components of $\mathfrak{C}\left(\mathfrak{g}_{1}\right)$ in the $\mathfrak{s l}_{n+m}$-case, which shows that this number can be arbitrarily large, see Proposition 4.4. In Section 5, we prove the reducibility of $\mathfrak{C}\left(\mathfrak{g}_{1}\right)$ for $\left(\mathcal{E}_{7}, \mathfrak{s o}_{12} \oplus \mathfrak{s} \mathfrak{l}_{2}\right)$ and $\left(\mathcal{E}_{8}, \mathcal{E}_{7} \oplus \mathfrak{s l}_{2}\right)$, using some ideas form the theory of principal nilpotent pairs.

Taking into account the previously known results and the results of this paper, one sees that there remain only three cases (two classical series and one symmetric pair for $\mathcal{E}_{7}$ ), where the the answer is not known. In particular, for series $\mathcal{A}$ and $\mathcal{B}$, the irreducibility problem is completely solved. In Section 6 we discuss possible approaches to the remaining cases.

Acknowledgements. This paper was written during our stay at the Max-Planck-Institut für Mathematik (Bonn). We are grateful to this institution for the warm hospitality and support.

\section{NilPOTENT ELEMENTS, SUB-SYMMETRIC PAIRS, AND COMMUTING VARIETIES}

In this section, we deal with semisimple and nilpotent elements in $\mathfrak{g}_{1}$ and their relations to the commuting variety.

Let $n$ be a non-negative integer. The set

$$
\mathfrak{g}_{1}^{(n)}=\left\{\xi \in \mathfrak{g}_{1} \mid \operatorname{dim}\left(G_{0} \xi\right)=n\right\}
$$

is locally closed. The irreducible components of the sets $\mathfrak{g}_{1}^{(n)}$ are called the $G_{0}$-sheets of $\mathfrak{g}_{1}$.

Lemma 1.1. Let $\mathcal{S}$ be a $G_{0}$-sheet of $\mathfrak{g}_{1}$ containing semisimple elements. Suppose that, for each semisimple $h \in \mathcal{S}$, we have $\left(\{h\} \times \mathfrak{g}_{1, h}\right) \subset \mathfrak{C}_{0}$. Then $\left(\{x\} \times \mathfrak{g}_{1, x}\right) \subset \mathfrak{C}_{0}$ for each $x \in \mathcal{S}$.

Proof. Let $x \in \mathcal{S}$. Since $\mathcal{S}$ contains semisimple elements, they form a dense open subset. Therefore, we can find a morphism $\gamma: \mathbb{k} \rightarrow \mathcal{S}$ such that $\gamma(0)=x$ and $\gamma(t)$ is semisimple for each $t \neq 0$. Then $\mathfrak{g}_{1, x}=\lim _{t \rightarrow 0} \mathfrak{g}_{1, \gamma(t)}$, where the limit is taken in an appropriate Grassmannian. For each $y \in \mathfrak{g}_{1, x}$, we can define elements $y(t) \in \mathfrak{g}_{1, \gamma(t)}$ such that $y=\lim _{t \rightarrow 0} y(t)$. Since $(x, y)=\lim _{t \rightarrow 0}(\gamma(t), y(t))$ and $(\gamma(t), y(t)) \in \mathfrak{C}_{0}$ for each $t \neq 0$, we conclude that $(x, y) \in \mathfrak{C}_{0}$.

Lemma 1.2. [6, Prop. 5] For any $x \in \mathfrak{g}_{1}$, we have $\operatorname{dim} \mathfrak{g}_{1, x}-\operatorname{dim} \mathfrak{g}_{0, x}=\operatorname{dim} \mathfrak{g}_{1}-\operatorname{dim} \mathfrak{g}_{0}$. 
Let $\mathfrak{N}\left(\mathfrak{g}_{1}\right)$ denote the set of all nilpotent elements in $\mathfrak{g}_{1}$. For any $e \in \mathfrak{N}\left(\mathfrak{g}_{1}\right)$, there is an $\mathfrak{s l}_{2}$-triple $\{e, f, h\}$ such that $f \in \mathfrak{g}_{1}$ and $h \in \mathfrak{g}_{0}[$ [6]. Such a triple is said to be normal. Recall that $e$ is called even if the eigenvalues of ad $h$ on $\mathfrak{g}$ are even.

Definition 1. An element $e \in \mathfrak{N}\left(\mathfrak{g}_{1}\right)$ is said to be $\sigma$-distinguished (in other notation, $\mathfrak{p}$ - or $(-1)$-distinguished) if $\mathfrak{g}_{1, e}$ contains no semisimple elements of $[\mathfrak{g}, \mathfrak{g}]$.

The following lemma appears in [15], but the proof given here is shorter.

Lemma 1.3. Suppose e $\in \mathfrak{N}\left(\mathfrak{g}_{1}\right)$ is even. Then e belongs to a $G_{0}$-sheet containing semisimple elements.

Proof. Let $(e, f, h)$ be a normal $\mathfrak{s l}_{2}$-triple. Since $e$ is even, we have $\operatorname{dim} \mathfrak{g}_{h}=\operatorname{dim} \mathfrak{g}_{e}$. Set $e(t):=e-t^{2} f$ for $t \in \mathbb{k}$. If $t \neq 0$, then $e(t)$ is semisimple and conjugate to $t h$. Therefore $\operatorname{dim} \mathfrak{g}_{e(t)}=\operatorname{dim} \mathfrak{g}_{h}=\operatorname{dim} \mathfrak{g}_{e}$ and $\operatorname{dim} \mathfrak{g}_{0, e(t)}=\operatorname{dim} \mathfrak{g}_{0, e}$ by Lemma 1.2. Clearly $e(0)=e=$ $\lim _{t \rightarrow 0} e(t)$ and the $G_{0}$-sheet containing $e$ contains also semisimple elements $e(t)$.

Let $h \in \mathfrak{g}_{1}$ be a semisimple element. Recall that $\mathfrak{C}\left(\mathfrak{g}_{1, h}\right)$ denotes for the commuting variety associated with the sub-symmetric pair $\left(\mathfrak{g}_{h}, \mathfrak{g}_{0, h}\right)$.

Theorem 1.4. Suppose that each $\sigma$-distinguished nilpotent element in $\mathfrak{g}_{1}$ is even and $\mathfrak{C}\left(\mathfrak{g}_{1, h}\right)$ is irreducible for each semisimple non-zero $h \in g_{1}$. Then $\mathfrak{C}\left(\mathfrak{g}_{1}\right)$ is irreducible.

Proof. Recall that $\mathfrak{C}_{0}=\overline{G_{0}(\mathfrak{c} \times \mathfrak{c})}$ is an irreducible component of $\mathfrak{C}\left(\mathfrak{g}_{1}\right)$. Following the original proof of Richardson [13] (see also [12, Sect. 2]), we show that $\mathfrak{C}\left(\mathfrak{g}_{1}\right)=\mathfrak{C}_{0}$. Let $(x, y) \in \mathfrak{C}\left(\mathfrak{g}_{1}\right)$.

(1) Suppose there is a semisimple element $h \in \mathfrak{g}_{1}$ such that $[h, x]=[h, y]=0$. This assumption is automatically satisfied if either $x$ or $y$ is semisimple. Moreover, if $x$ (or $y$ ) is not nilpotent and $x=x_{s}+x_{n}$ is the Jordan decomposition, then $x_{s} \in \mathfrak{g}_{1}$ and $\left[x_{s}, x\right]=0$, $\left[x_{s}, y\right]=0$.

Consider the sub-symmetric pair $\left(\mathfrak{g}_{h}, \mathfrak{g}_{0, h}\right)$. Replacing $\mathfrak{c}$ with a conjugate Cartan subspace, we may assume that $h \in \mathfrak{c}$. Then $\mathfrak{c}$ is a Cartan subspace of $\mathfrak{g}_{1, h}$. By the assumption, $\mathfrak{C}\left(\mathfrak{g}_{1, h}\right)$ is irreducible. Therefore, $(x, y) \in \overline{G_{0, h}(\mathfrak{c} \times \mathfrak{c})}$ and hence $(x, y) \in \mathfrak{C}_{0}$.

(2) Now we may assume that both $x$ and $y$ are nilpotent. Suppose first that there is a semisimple element $h \in \mathfrak{g}_{1}$ such that $[x, h]=0$. Then $(x,(1-t) y+t h) \in \mathfrak{C}\left(\mathfrak{g}_{1}\right)$ for each $t \in \mathbb{k}$ and $(1-t) y+t h$ is nilpotent only for a finite number of $t^{\prime} s$. Therefore, by part (1), one has $(x,(1-t) y+t h) \in \mathfrak{C}_{0}$ for almost all $t$. Since $y=\lim _{t \rightarrow 0}((1-t) y+t h)$, we get $(x, y) \in \mathfrak{C}_{0}$.

(3) It remains to handle the case in which both $x$ and $y$ are $\sigma$-distinguished nilpotent elements. Then both $x$ and $y$ are even and by Lemma 1.3, $x$ belongs to a $G_{0}$-sheet containing 
semisimple elements. According to part (1), the assumptions of Lemma 1.1 are satisfied and it follows that $(x, y) \in \mathfrak{C}_{0}$.

In order to effectively apply Theorem 1.4, we need a description of possible subsymmetric pairs. To this end, we recall the notion of the Satake diagram of a symmetric pair $\left(\mathfrak{g}, \mathfrak{g}_{0}\right)$, where $\mathfrak{g}$ is semisimple. Roughly speaking, it is the Dynkin diagram of $\mathfrak{g}$, where each node is either black or white and some pairs of white nodes are connected by a (new) arrow. More precisely, let $\mathfrak{t}$ be a $\sigma$-stable Cartan subalgebra of $\mathfrak{g}$ containing $\mathfrak{c}$ and let $\Delta$ be the root system of $(\mathfrak{g}, \mathfrak{t})$. Since $\mathfrak{t}$ is $\sigma$-stable, $\sigma$ acts on $\Delta$. It is possible to choose the set of positive roots, $\Delta^{+}$, such that if $\beta \in \Delta^{+}$and $\beta(\mathfrak{c}) \neq 0$, then $\sigma(\beta) \in-\Delta^{+}$. Let $\Pi$ be the corresponding set of simple roots and $\alpha \in \Pi$. Then the node corresponding to $\alpha$ is black if and only if $\alpha(\mathfrak{c})=0$. Two different white nodes corresponding to $\alpha_{i}, \alpha_{j} \in \Pi$ are connected by an arrow if and only if $\left.\alpha_{i}\right|_{\mathfrak{c}}=\left.\alpha_{j}\right|_{\mathfrak{c}}$. The latter is equivalent to that $\sigma\left(\alpha_{i}\right)=-\alpha_{j}$. Looking at the Satake diagram, one immediately reads off many properties of a symmetric pair under consideration. For instance,

$$
\operatorname{rk}\left(\mathfrak{g}, \mathfrak{g}_{0}\right)=\text { (number of white nodes) - (number of arrows), }
$$

and the centraliser of a generic element of $\mathfrak{c}$ is the Levi subalgebra of $\mathfrak{g}$ whose semisimple part is given by the subdiagram consisting of all black nodes.

Let $S$ be a Satake diagram. Then $S^{\prime}$ is a subdiagram of $S$ if $S^{\prime}$ is obtained from $S$ by repeating the following procedure. Namely, one can remove one white node, if it is not connected by an arrow; or one can remove a pair of nodes connected by an arrow.

Let $h \in \mathfrak{g}_{1}$ be semisimple. Set $\mathfrak{p}=\left[\mathfrak{g}_{h}, \mathfrak{g}_{h}\right]$ and $\mathfrak{p}_{i}=\mathfrak{g}_{i} \cap \mathfrak{p}$. Then $\left(\mathfrak{p}, \mathfrak{p}_{0}\right)$ is said to be a reduced sub-symmetric pair of $\left(\mathfrak{g}, \mathfrak{g}_{0}\right)$. Clearly, $\mathfrak{C}\left(\mathfrak{g}_{1, h}\right)$ is irreducible if and only if $\mathfrak{C}\left(\mathfrak{p}_{1}\right)$ is irreducible. So, it is enough to describe all reduced sub-symmetric pairs.

Proposition 1.5. A symmetric pair $\left(\mathfrak{p}, \mathfrak{p}_{0}\right)$ is a reduced sub-symmetric pair of $\left(\mathfrak{g}, \mathfrak{g}_{0}\right)$ if and only if $S_{\mathfrak{p}}$, the Satake diagram of $\left(\mathfrak{p}, \mathfrak{p}_{0}\right)$, is a sub-diagram of $S_{\mathfrak{y}}$, the Satake diagram of $\left(\mathfrak{g}, \mathfrak{g}_{0}\right)$.

Proof. Without loss of generality, we may assume that $h \in \mathfrak{c}$. Then $\alpha(h)=0$ if the node corresponding to $\alpha \in \Pi$ is black. Since we are only interested in possible types of centralisers, we may assume that $h$ lies in the $\mathbb{Q}$-form $\mathfrak{c}_{\mathbb{Q}}$. Here $\mathfrak{c}_{\mathbb{Q}}$ is the $\mathbb{Q}$-span of all restricted roots $\left.\beta\right|_{\mathfrak{c}}, \beta \in \Delta$, and $\mathfrak{c}$ is identified with $\mathfrak{c}^{*}$ using the restriction of the Killing form. Moreover, using the action of the little Weyl group on $\mathfrak{c}_{\mathbb{Q}}$, we may assume that $\alpha(h)>0$ if the node corresponding to $\alpha \in \Pi$ is white. Then the Dynkin diagram $D_{\mathfrak{p}}$ of $\mathfrak{p}$ is a subdiagram of $D_{\mathfrak{g}}$ consisting of all $\alpha \in \Pi$ such that $\alpha(h)=0$. In particular, $D_{\mathfrak{p}}$ contains all black nodes of $S_{\mathfrak{g}}$.

Set $\mathfrak{c}_{\mathfrak{p}}:=\mathfrak{c} \cap \mathfrak{p}$. It is a Cartan subspace of $\mathfrak{p}_{1}$ and $\mathfrak{c}=\mathfrak{c}_{\mathfrak{p}} \oplus \mathfrak{z}_{1}$, where $\mathfrak{z}_{1}$ lies in the centre of $\mathfrak{g}_{h}$. If $\alpha_{i}$ is a simple root of $\mathfrak{p}$, then $\alpha_{i}\left(\mathfrak{z}_{1}\right)=0$ and $\alpha_{i}\left(\mathfrak{c}_{\mathfrak{p}}\right)=0$ if and only if $\alpha_{i}(\mathfrak{c})=0$. Hence the nodes do not change their colours. Suppose $\alpha_{i}$ and $\alpha_{j}$ are connected by an arrow in 
$S_{\mathfrak{g}}$. Then $\left(\alpha_{i}-\alpha_{j}\right)(\mathfrak{c})=0$. In particular, $\alpha_{i}(h)=0$ if and only if $\alpha_{j}(h)=0$. If $\alpha_{i}$ and $\alpha_{j}$ belong to $D_{\mathfrak{p}}$, then they are still connected by an arrow, since $\mathfrak{c}_{\mathfrak{p}} \subset \mathfrak{c}$. Conversely, if $\alpha_{i}, \alpha_{j}$ are simple roots of $\mathfrak{p}$ and they are connected by an arrow of $S_{\mathfrak{p}}$, then $\left(\alpha_{i}-\alpha_{j}\right)\left(\mathfrak{c}_{\mathfrak{p}}\right)=0$ and $\left(\alpha_{i}-\alpha_{j}\right)\left(\mathfrak{z}_{1}\right)=0$. Therefore $\left(\alpha_{i}-\alpha_{j}\right)(\mathfrak{c})=0$, and $\alpha_{i}, \alpha_{j}$ are connected by an arrow in $S_{\mathfrak{g}}$. Thus, the Satake diagram of any reduced sub-symmetric pair is a subdiagram of $S_{\mathfrak{g}}$.

Conversely, let $S^{\prime}$ be a subdiagram of $S_{\mathfrak{g}}$ in the sense of the above definition. Consider the subspace $\mathfrak{c}^{\prime} \subset \mathfrak{c}$, where all simple roots corresponding to the nodes from $S_{\mathfrak{g}} \backslash S^{\prime}$ vanish. Then, for a generic $h \in \mathfrak{c}^{\prime}$, we obtain the reduced sub-symmetric pair whose Satake diagram is $S^{\prime}$.

2. $\mathfrak{C}\left(\mathfrak{g}_{1}\right)$ IS IRREDUCIBLE FOR $\left(\mathfrak{g}, \mathfrak{g}_{0}\right)=\left(\mathfrak{s o}_{n+m}, \mathfrak{s o}_{n} \oplus \mathfrak{s} \mathfrak{o}_{m}\right)$

In this section $G=\mathrm{SO}_{n+m}, \mathfrak{g}=\mathfrak{s o}_{n+m}$, and $\mathfrak{g}_{0}=\mathfrak{s o}_{n} \oplus \mathfrak{s o _ { m }}$. Let $V=\mathbb{k}^{n+m}$ be a vector space of the defining representation of $\mathfrak{g}$. Then we have a $G_{0}$-invariant decomposition $V=V_{a} \oplus V_{b}$, where $V_{a}=\mathbb{k}^{n}, V_{b}=\mathbb{k}^{m}$ and $\mathfrak{g}_{1} \cong \mathbb{k}^{n} \otimes \mathbb{k}^{m}$ as a $G_{0}$-module. Let ( , ) denote the non-degenerate symmetric $G$-invariant bilinear form on $V$. In order to apply Theorem 1.4, we need a description of semisimple and nilpotent elements in $\mathfrak{g}_{1}$.

Lemma 2.1. Let $h \in \mathfrak{g}_{1}$ be a semisimple element. Then the symmetric pair $\left(\mathfrak{g}_{h}, \mathfrak{g}_{0, h}\right)$ is a direct $\operatorname{sum}\left(\bigoplus_{i=1}^{r}\left(\mathfrak{g l}_{k_{i}}, \mathfrak{s o}_{k_{i}}\right)\right) \oplus\left(\mathfrak{s o}_{n+m-2 k}, \mathfrak{s o}_{n-k} \oplus \mathfrak{s o}_{m-k}\right)$, where $k=\sum_{i} k_{i}$.

Proof. Let $v_{\lambda} \in V$ be an eigenvector of $h$ such that $h \cdot v_{\lambda}=\lambda v_{\lambda}$ and $\lambda \neq 0$. Since $h$ preserves the symmetric form $\left(\right.$, ), i.e., $\left(h \cdot v_{\lambda}, v_{\lambda}\right)+\left(v_{\lambda}, h \cdot v_{\lambda}\right)=0$, we have $\left(v_{\lambda}, v_{\lambda}\right)=0$. Also, if $h \cdot v=\lambda v, h \cdot w=\mu w$, then $(v, w)=0$ unless $\lambda=-\mu$. Let $\left\{ \pm \lambda_{i}, 0 \mid i=1, \ldots, r\right\}$ be the set of the eigenvalues of $h$. Then there is an orthogonal $h$-invariant decomposition

$$
V=\left(V_{\lambda_{1}} \oplus V_{-\lambda_{1}}\right) \oplus \ldots \oplus\left(V_{\lambda_{r}} \oplus V_{-\lambda_{r}}\right) \oplus V_{0} .
$$

Here each $V_{\lambda_{i}}$ is an isotropic subspace, $\left(V_{\lambda_{i}}, V_{\lambda_{j}}\right)=0$ unless $\lambda_{i}+\lambda_{j}=0$, and $\left(V_{0}, V_{ \pm \lambda_{i}}\right)=0$ for each $\lambda_{i}$. Therefore $\mathfrak{g}_{h} \subset\left(\bigoplus_{i=1}^{r} \mathfrak{s o}\left(V_{\lambda_{i}} \oplus V_{-\lambda_{i}}\right)\right) \oplus \mathfrak{s o}\left(V_{0}\right)$. More precisely, if $\operatorname{dim} V_{\lambda_{i}}=k_{i}$ and $k=\sum_{i} k_{i}$, then $\mathfrak{g}_{h}=\left(\bigoplus_{i} \mathfrak{g l}_{k_{i}}\right) \oplus \mathfrak{s o}_{n+m-2 k}$.

Now it remains to describe $\mathfrak{g}_{0, h}=\left(\mathfrak{g}_{h}\right)^{\sigma}$. We may assume that $\sigma$ is a conjugation by a diagonal matrix $A \in \mathrm{O}_{n+m}$ such that $\left.A\right|_{V_{a}}=-\mathrm{id}$ and $\left.A\right|_{V_{b}}=\mathrm{id}$. Since $\sigma(h)=-h$, we have $A \cdot V_{\lambda_{i}}=V_{-\lambda_{i}}$ and $A \cdot V_{0}=V_{0}$. Moreover, $A$ determines a non-degenerate symmetric form $(,)_{A}$ on each $V_{\lambda_{i}}$ by the formula $(v, w)_{A}=(v, A \cdot w)$. Therefore, each $\mathfrak{s o}\left(V_{\lambda_{i}} \oplus V_{-\lambda_{i}}\right)$ is $\sigma$-invariant, $\left(\mathfrak{s o}\left(V_{\lambda_{i}} \oplus V_{-\lambda_{i}}\right)\right)^{\sigma} \cong \mathfrak{s o}_{k_{i}} \oplus \mathfrak{s o}_{k_{i}}$, and $\left(\mathfrak{g l}_{k_{i}}\right)^{\sigma}=\mathfrak{s o}_{k_{i}}$. Finally, the restriction $\left.A\right|_{V_{0}}$ has signature $(n-k, m-k)$. Thus $\left(\mathfrak{s o}_{n+m-2 k}\right)^{\sigma}=\mathfrak{s o}_{n-k} \oplus \mathfrak{s o}_{m-k}$.

Note that this result can also be deduced from Proposition 1.5 , 
Recall several standard facts concerning nilpotent elements in $\mathfrak{g l}(V)$. Suppose $e \in \mathfrak{g l}(V)$ is nilpotent and $m=\operatorname{dim} \operatorname{Ker}(e)$. By the theory of Jordan normal form, there are vectors $w_{1}, \ldots, w_{m} \in V$ and non-negative integers $d_{1}, \ldots, d_{m}$ such that $e^{d_{i}} \cdot w_{i}=0$ and $\left\{e^{s} \cdot w_{i} \mid 1 \leqslant i \leqslant m, 0 \leqslant s<d_{i}\right\}$ is a basis for $V$. Let $V[i] \subset V$ be the linear span of $\left\{w_{i}, e \cdot w_{i}, \ldots, e^{d_{i}-1} \cdot w_{i}\right\}$. The spaces $\{V[i]\}$ are called the Jordan (or cyclic) spaces of $e$, and $V=\oplus_{i=1}^{m} V[i]$.

Lemma 2.2. Suppose $e \in \mathfrak{N}\left(\mathfrak{g}_{1}\right)$. Then the cyclic vectors $\left\{w_{i}\right\}_{i=1}^{m}$ and hence the cyclic spaces $\{V[i]\}$ 's can be chosen such that the following properties are satisfied:

(i) there is an involution $i \mapsto i^{*}$ on the set $\{1, \ldots, m\}$ such that: $d_{i}=d_{i^{*}} ; i=i^{*}$ if and only if $\operatorname{dim} V[i]$ is odd; $(V[i], V[j])=0$ if $i \neq j^{*}$;

(ii) $\sigma\left(w_{i}\right)= \pm w_{i}$.

Proof. Part (i) is a standard property of the nilpotent orbits in $\mathfrak{s o}(V)$, see, for example, [1, Sect. 5.1] or [5, Sect. 1]. Then part (ii) says that in the presence of the involution $\sigma$ cyclic vectors for $e \in \mathfrak{N}\left(\mathfrak{g}_{1}\right)$ can be chosen to be $\sigma$-eigenvectors, see [8, Prop. 2].

For each $e \in \mathfrak{N}\left(\mathfrak{g}_{1}\right)$, we choose cyclic vectors $\left\{w_{i}\right\}$ as prescribed by Lemma 2.2. Let us say that $e^{s} \cdot w_{i}$ is of type $a$ if $\sigma\left(e^{s} \cdot w_{i}\right)=-e^{s} \cdot w_{i}$, i.e., $e^{s} \cdot w_{i} \in V_{a}$; and $e^{s} \cdot w_{i}$ is of type $b$ if $e^{s} \cdot w_{i} \in V_{b}$. Since $\sigma(e)=-e$, if $e^{s} \cdot w_{i} \in V_{a}$, then $e^{s+1} \cdot w_{i} \in V_{b}$ and vice versa. Therefore each string $\left(w_{i}, e \cdot w_{i}, \ldots, e^{d_{i}-1} \cdot w_{i}\right)$ has one of the following types: $a b a \ldots a b, \quad b a b \ldots b a, \quad a b a \ldots b a, \quad b a b \ldots a b$.

Proposition 2.3. Every $\sigma$-distinguished element $e \in \mathfrak{N}\left(\mathfrak{g}_{1}\right)$ is even.

Proof. Suppose $e \in \mathfrak{N}\left(\mathfrak{g}_{1}\right)$ is $\sigma$-distinguished and the cyclic spaces $\{V[i]\}$ are chosen as prescribed by Lemma 2.2. Assume that $\operatorname{dim} V[i]$ is even for some $i$. By [8, Prop. 2], if $V[i]$ is of type $a b a \ldots a b$, then $V\left[i^{*}\right]$ is necessarily of type $b a b \ldots b a$, and vice versa; i.e., if $\sigma\left(w_{i}\right)=$ $\mp w_{i}$, then $\sigma\left(w_{i^{*}}\right)= \pm w_{i^{*}}$. It is easily seen that $\mathfrak{s o}\left(V[i] \oplus V\left[i^{*}\right]\right) \cap \mathfrak{g}_{e}$ contains a $\sigma$-stable subalgebra $\mathfrak{l}$ that is isomorphic to $\mathfrak{s l}\left(\mathbb{k} w_{i} \oplus \mathbb{k} w_{i^{*}}\right) \cong \mathfrak{s l}_{2}$. More precisely, any unimodular transformation of $\mathbb{k} w_{i} \oplus \mathbb{k} w_{i^{*}}$ can be uniquely extended to an element of $\mathfrak{s o}\left(V[i] \oplus V\left[i^{*}\right]\right) \cap \mathfrak{g}_{e}$. Since the restriction of $\sigma$ to $\mathfrak{l}$ is non-trivial, $\mathfrak{l}_{1}=\mathfrak{l} \cap \mathfrak{g}_{1} \subset \mathfrak{g}_{e}$ contains semisimple elements. This means that such $e$ is not $\sigma$-distinguished. Hence, all $V[i]$ are odd-dimensional. Since a nilpotent element of $\mathfrak{s o}_{N}$ is even if and only if all the numbers $\operatorname{dim} V[i]$ have the same parity, we are done.

Theorem 2.4. For $\left(\mathfrak{g}, \mathfrak{g}_{0}\right)=\left(\mathfrak{s o}_{n+m}, \mathfrak{s \mathfrak { s } _ { n }} \oplus \mathfrak{s o}_{m}\right)$, the commuting variety $\mathfrak{C}\left(\mathfrak{g}_{1}\right)$ is irreducible.

Proof. Let $\mathfrak{c} \subset \mathfrak{g}_{1}$ be a Cartan subspace. We argue by induction on $\operatorname{dim} \mathfrak{c}$. The base of induction is the rank 1 case $\left(\mathfrak{s o}_{n+1}, \mathfrak{s o}_{n}\right)$, where the irreducibility of $\mathfrak{C}\left(\mathfrak{g}_{1}\right)$ is proved in [12], [14]. 
Let $h \neq 0$ be a semisimple element of $\mathfrak{g}_{1}$. By Lemma 2.1. $\left(\mathfrak{g}_{h}, \mathfrak{g}_{0, h}\right)=\left(\bigoplus_{i=1}^{r}\left(\mathfrak{g l}_{k_{i}}, \mathfrak{s o}_{k_{i}}\right)\right) \oplus$ $\left(\mathfrak{s o}_{n+m-2 k}, \mathfrak{s o}_{n-k} \oplus \mathfrak{s o}_{m-k}\right)$. Each pair $\left(\mathfrak{g l}_{k_{i}}, \mathfrak{s o}_{k_{i}}\right)$ is a symmetric pair of maximal rank, hence, the corresponding commuting variety is irreducible, see [10, (3.5)(1)]. Clearly, the commuting variety corresponding to a direct sum of symmetric pairs is the product of the commuting varieties corresponding to the summands. Therefore, using the inductive hypothesis, we conclude that $\mathfrak{C}\left(\mathfrak{g}_{1, h}\right)$ is irreducible. Taking into account Proposition 2.3, we see that the assumptions of Theorem 1.4 are satisfied and $\mathfrak{C}\left(\mathfrak{g}_{1}\right)$ is irreducible.

\section{IRREDUCIBILITY OF $\mathfrak{C}\left(\mathfrak{g}_{1}\right)$ FOR SYMMETRIC PAIRS WITH $\operatorname{dim} \mathfrak{g}_{1}>\operatorname{dim} \mathfrak{g}_{0}$}

In this section we show that the method of proving irreducibility of $\mathfrak{C}\left(\mathfrak{g}_{1}\right)$ in the maximal rank case [10] actually applies in a more general setting of symmetric pairs with $\operatorname{dim} \mathfrak{g}_{1}>\operatorname{dim} \mathfrak{g}_{0}$. Practically, this yields the irreducibility for two new pairs: $\left(\mathfrak{s l}_{2 n}, \mathfrak{s l}_{n} \oplus \mathfrak{s} \mathfrak{l}_{n} \oplus \mathfrak{t}_{1}\right)$ and $\left(\mathcal{E}_{6}, \mathfrak{s l}_{6} \oplus \mathfrak{s l}_{2}\right)$.

Recall that $\left(\mathfrak{g}, \mathfrak{g}_{0}\right)$ is of maximal rank if and only if the Satake diagram has no new arrows and all the nodes are white.

Lemma 3.1. Suppose all nodes of the Satake diagram of $\left(\mathfrak{g}, \mathfrak{g}_{0}\right)$ are white and there is at least one that is not connected by an arrow with any of the others. Then $\operatorname{dim} \mathfrak{g}_{1}>\operatorname{dim} \mathfrak{g}_{0}$.

Proof. Choose a semisimple element $h \in \mathfrak{g}_{1}$ such that $\mathfrak{g}_{1, h}=\mathfrak{c}$ is a Cartan subspace. Then $\left(\mathfrak{g}_{0}\right)_{h}$ coincides with the centraliser of $\mathfrak{c}$ in $\mathfrak{g}_{0}$. Therefore the structure of $\mathfrak{g}_{0, h}$ can be read off from the Satake diagram. In our case $\mathfrak{g}_{0, h}$ is a torus and $\operatorname{dim} \mathfrak{g}_{0, h}=\operatorname{rk} \mathfrak{g}-\operatorname{dim} \mathfrak{c}<(\operatorname{rkg} \mathfrak{g}) / 2$. Hence $\operatorname{dim} \mathfrak{g}_{0, h}<\operatorname{dim} \mathfrak{c}$, and $\operatorname{dim} \mathfrak{g}_{0}<\operatorname{dim} \mathfrak{g}_{1}$ in view of Lemma1.2.

Another simple observation is that $\mathfrak{g}_{1} \times \mathfrak{g}_{1} \cong T^{*}\left(\mathfrak{g}_{1}\right)$ and the commutator morphism $\psi: \mathfrak{g}_{1} \times \mathfrak{g}_{1} \rightarrow \mathfrak{g}_{0}$ is the moment map for the natural Hamiltonian action of $G_{0}$ on $T^{*}\left(\mathfrak{g}_{1}\right)$. Therefore $\psi$ is dominant if and only if the generic stabiliser for the diagonal action of $G_{0}$ on $\mathfrak{g}_{1} \times \mathfrak{g}_{1}$ is finite. This is always true if the centraliser of $\mathfrak{c}$ in $\mathfrak{g}_{0}$ is a torus, i.e., the Satake diagram contains no black nodes.

Lemma 3.2. Suppose $\operatorname{dim} \mathfrak{g}_{1}>\operatorname{dim} \mathfrak{g}_{0}, \psi$ is dominant, and $\mathfrak{C}\left(\mathfrak{g}_{1, h}\right)$ is irreducible for all non-zero semisimple $h \in \mathfrak{g}_{1}$. Then $\mathfrak{C}\left(\mathfrak{g}_{1}\right)$ is irreducible.

Proof. The dominance of $\psi$ implies that if $\psi^{-1}(\xi) \neq \varnothing$, then all irreducible components of $\psi^{-1}(\xi)$ are of dimension $\geqslant 2 \operatorname{dim} \mathfrak{g}_{1}-\operatorname{dim} \mathfrak{g}_{0}$. Since $2 \operatorname{dim} \mathfrak{g}_{1}-\operatorname{dim} \mathfrak{g}_{0}>\operatorname{dim} \mathfrak{g}_{1}$, all irreducible components of $\psi^{-1}(0)=\mathfrak{C}\left(\mathfrak{g}_{1}\right)$ are of dimension $>\operatorname{dim} \mathfrak{g}_{1}$.

Let $x \in \mathfrak{g}_{1}$. Then $\overline{G_{0}\left(\{x\}, \mathfrak{g}_{1, x}\right)}$ is an irreducible subvariety of $\mathfrak{C}\left(\mathfrak{g}_{1}\right)$ of dimension $\operatorname{dim} \mathfrak{g}_{1}$. If $x$ is not nilpotent, then $\overline{G_{0}\left(\{x\}, \mathfrak{g}_{1, x}\right)} \subset \mathfrak{C}_{0}$, see proof of Theorem 1.4 part (1). As is well known, $G_{0}$ has finitely many nilpotent orbits on $\mathfrak{g}_{1}$. Hence $\mathfrak{C}\left(\mathfrak{g}_{1}\right)$ is a union of $\mathfrak{C}_{0}$ and 
finite many irreducible varieties of dimension $\operatorname{dim} \mathfrak{g}_{1}$. Since each irreducible component of $\mathfrak{C}\left(\mathfrak{g}_{1}\right)$ has dimension greater than $\operatorname{dim} \mathfrak{g}_{1}, \mathfrak{C}\left(\mathfrak{g}_{1}\right)$ must be irreducible.

Theorem 3.3. The commuting varieties associated with $\left(\mathfrak{s l}_{2 n}, \mathfrak{s l}_{n} \oplus \mathfrak{s l}_{n} \oplus \mathfrak{t}_{1}\right)$ and $\left(\mathcal{E}_{6}, \mathfrak{s l}_{6} \oplus \mathfrak{s l}_{2}\right)$ are irreducible.

Proof. First consider the classical case. We argue by induction on $n$. If $n=1$, then $\left(\mathfrak{g}, \mathfrak{g}_{0}\right)=$ $\left(\mathfrak{s o}_{3}, \mathfrak{s o}_{2}\right)$ and the corresponding commuting variety is irreducible. In order to make an induction step, we use Lemma 3.2. Here $\operatorname{dim} \mathfrak{g}_{1}=2 n^{2}$, $\operatorname{dim} \mathfrak{g}_{0}=2 n^{2}-1$. The generic stabiliser for the action of $G_{0}$ on $\mathfrak{g}_{1}$ is an $(n-1)$-dimensional torus. It remains to show that $\mathfrak{C}\left(\mathfrak{g}_{1, h}\right)$ is irreducible for all non-zero semisimple $h \in \mathfrak{g}_{1}$.

Let $h \in \mathfrak{g}_{1}$ be semisimple. Using either Lemma 1.5 or the same argument as in Lemma 2.1. one can show that the semisimple part of $\left(\mathfrak{g}_{h}, \mathfrak{g}_{0, h}\right)$ is a direct sum of $\bigoplus_{i=1}^{r}\left(\mathfrak{s l}_{k_{i}} \oplus \mathfrak{s l}_{k_{i}}, \mathfrak{s l}_{k_{i}}\right)$ and $\left(\mathfrak{s l}_{2 n-2 k}, \mathfrak{s l}_{n-k} \oplus \mathfrak{s l}_{n-k} \oplus \mathfrak{t}_{1}\right)$. Therefore $\mathfrak{C}\left(\mathfrak{g}_{1, h}\right)$ is irreducible and we are done.

Consider now the exceptional case. The Satake diagram of $\left(\mathcal{E}_{6}, \mathfrak{s l}_{6} \oplus \mathfrak{s l}_{2}\right)$ is:

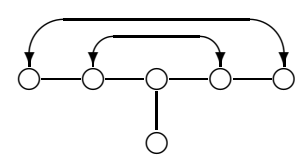

It contains no black nodes, hence $\psi$ is dominant. There are two nodes which are not connected by an arrow with others, hence, by Lemma 3.1, $\operatorname{dim} \mathfrak{g}_{1}>\operatorname{dim} \mathfrak{g}_{0}$. In fact, $\operatorname{dim} \mathfrak{g}_{1}=40$ and $\operatorname{dim} \mathfrak{g}_{0}=38$. To apply Lemma 3.2, one has to find all Satake subdiagrams and verify that the associated commuting varieties are irreducible.

If a Satake diagram is disconnected as a graph, then the corresponding symmetric pair is a direct sum of two non-trivial symmetric pairs. Since the commuting variety associated with a direct sum of symmetric pairs is a product of the commuting varieties associated with the summands, we can restrict ourselves to connected subdiagrams. There are seven of them.
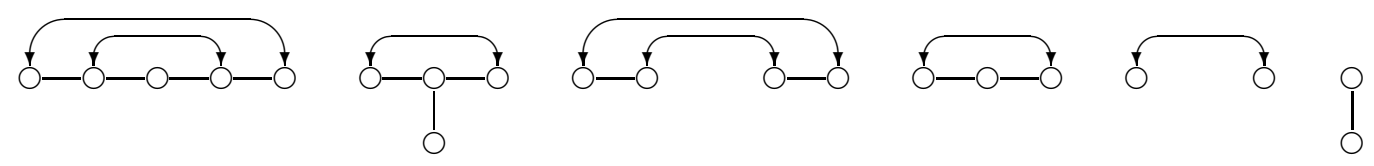

These diagrams correspond to the following symmetric pairs:

$$
\begin{aligned}
& \left(\mathfrak{s l}_{6}, \mathfrak{s l}_{3} \oplus \mathfrak{s l}_{3} \oplus \mathfrak{t}_{1}\right),\left(\mathfrak{s o}_{8}, \mathfrak{s o}_{5} \oplus \mathfrak{s o}_{3}\right),\left(\mathfrak{s l}_{3} \oplus \mathfrak{s l}_{3}, \mathfrak{s l}_{3}\right), \\
& \left(\mathfrak{s l}_{4}, \mathfrak{s l}_{2} \oplus \mathfrak{s l}_{2} \oplus \mathfrak{t}_{1}\right)=\left(\mathfrak{s o}_{6}, \mathfrak{s o}_{4} \oplus \mathfrak{s o}_{2}\right),\left(\mathfrak{s l}_{2} \oplus \mathfrak{s l}_{2}, \mathfrak{s l}_{2}\right),\left(\mathfrak{s l}_{3}, \mathfrak{s o}_{3}\right),\left(\mathfrak{s l}_{2}, \mathfrak{s o}_{2}\right) .
\end{aligned}
$$

For all of them the irreducibility of $\mathfrak{C}\left(\mathfrak{g}_{1}\right)$ is already established. 


\section{SHORT $\mathbb{Z}$-GRADINGS AND REDUCIBLE COMMUTING VARIETIES}

Let $\mathfrak{g}=\mathfrak{g}(-1) \oplus \mathfrak{g}(0) \oplus \mathfrak{g}(1)$ be a short $\mathbb{Z}$-grading of a reductive Lie algebra $\mathfrak{g}$. Set $\mathfrak{g}_{0}:=\mathfrak{g}(0)$. Then $\left(\mathfrak{g}, \mathfrak{g}_{0}\right)$ is a symmetric pair with $\mathfrak{g}_{1}=\mathfrak{g}(-1) \oplus \mathfrak{g}(1)$. Let $\mathfrak{c} \subset \mathfrak{g}_{1}$ be a Cartan subspace and let $\mathfrak{c}( \pm 1)$ denote the image of $\mathfrak{c}$ under the projection to $\mathfrak{g}( \pm 1)$. As $\mathfrak{c}$ consists of semisimple elements, we have $\mathfrak{c} \cap \mathfrak{g}( \pm 1)=0$ and $\operatorname{dim} \mathfrak{c}=\operatorname{dim} \mathfrak{c}(1)=\operatorname{dim} \mathfrak{c}(-1)$. Below, we consider the diagonal $G_{0}$-action on $\mathfrak{g}(1) \times \mathfrak{g}(1)$.

Lemma 4.1. Suppose $\mathfrak{C}\left(\mathfrak{g}_{1}\right)$ is irreducible. Then

$$
\overline{G_{0}(\mathfrak{c}(1) \times \mathfrak{c}(1))}=\mathfrak{g}(1) \times \mathfrak{g}(1) .
$$

Proof. Notice that $\mathfrak{g}(1)$ is a commutative subalgebra, hence $\mathfrak{g}(1) \times \mathfrak{g}(1) \subset \mathfrak{C}\left(\mathfrak{g}_{1}\right)$. Since $\mathfrak{c} \subset \mathfrak{c}(-1) \oplus \mathfrak{c}(1)$, we have

$$
\overline{G_{0}(\mathfrak{c} \times \mathfrak{c})} \subset \overline{G_{0}(\mathfrak{c}(-1) \times \mathfrak{c}(1) \times \mathfrak{c}(-1) \times \mathfrak{c}(1))} \subset \overline{G_{0}(\mathfrak{c}(-1) \times \mathfrak{c}(-1))} \times \overline{G_{0}(\mathfrak{c}(1) \times \mathfrak{c}(1))} .
$$

Hence $\overline{G_{0}(\mathfrak{c} \times \mathfrak{c})} \cap(\mathfrak{g}(1) \times \mathfrak{g}(1)) \subset \overline{G_{0}(\mathfrak{c}(1) \times \mathfrak{c}(1))}$. If $\mathfrak{C}\left(\mathfrak{g}_{1}\right)$ is irreducible, then $\overline{G_{0}(\mathfrak{c} \times \mathfrak{c})} \cap(\mathfrak{g}(1) \times \mathfrak{g}(1))=\mathfrak{g}(1) \times \mathfrak{g}(1)$, and we get equality $\left(\boldsymbol{\uparrow}_{1}\right)$.

Similarly, one can consider condition $\left(\mathbf{A}_{-1}\right)$ with $\mathfrak{c}(-1)$ in place of $\mathfrak{c}(1)$, etc. It is easily seen that $\left(\mathbf{p}_{1}\right)$ is satisfied if and only if $\left(\boldsymbol{\omega}_{-1}\right)$ is. For this reason, this common condition is denoted by $(\mathbf{\uparrow})$.

Corollary 4.2. If condition ( $)$ is not satisfied, then $\mathfrak{C}\left(\mathfrak{g}_{1}\right)$ has at least three irreducible components.

Proof. Along with the standard component $\mathfrak{C}_{0}$, we have at least two other components determined by $\mathfrak{g}(1) \times \mathfrak{g}(1)$ and $\mathfrak{g}(-1) \times \mathfrak{g}(-1)$.

Now we give three examples of symmetric pairs arising from short $\mathbb{Z}$-gradings such that condition $(\mathbf{Q})$ is not satisfied.

Example 4.3. $\left(\mathfrak{g}, \mathfrak{g}_{0}\right)=\left(\mathfrak{s l}_{n+m}, \mathfrak{s l}_{n} \oplus \mathfrak{s l}_{m} \oplus \mathfrak{t}_{1}\right)$.

It will be easier to work with the symmetric pair $\left(\mathfrak{g l}_{n+m}, \mathfrak{g l}_{n} \oplus \mathfrak{g l}_{m}\right)$ having the same commuting variety. We use the following matrix model. Let $V$ be an $(n+m)$-dimensional vector space and $\mathfrak{g}=\mathfrak{g l}(V)$. Let $V=V_{a} \oplus V_{b}$ be a vector space decomposition with $\operatorname{dim} V_{a}=n$ and $\operatorname{dim} V_{b}=m$. Then $\mathfrak{g}(1)=\operatorname{Hom}\left(V_{a}, V_{b}\right), \mathfrak{g}(-1)=\operatorname{Hom}\left(V_{b}, V_{a}\right)$, and $\mathfrak{g}(0)=\operatorname{Hom}\left(V_{a}, V_{a}\right) \oplus \operatorname{Hom}\left(V_{b}, V_{b}\right) \simeq \mathfrak{g l}_{n} \oplus \mathfrak{g l}_{m}$. The corresponding involution $\sigma$ is the inner automorphism determined by the matrix $A \in \mathrm{GL}(V)$ such that $\left.A\right|_{V_{a}}=-\mathrm{id},\left.A\right|_{V_{b}}=\mathrm{id}$. In the matrix form, every $\xi \in \mathfrak{g}_{1}$ is depicted as follows: $\xi=\left(\begin{array}{cc}0 & \xi(-1) \\ \xi(1) & 0\end{array}\right)$, where $\xi(1) \in \operatorname{Hom}\left(V_{a}, V_{b}\right)$ is an $m \times n$ matrix and $\xi(-1) \in \operatorname{Hom}\left(V_{b}, V_{a}\right)$ is an $n \times m$ matrix. 
Assume that $n<m$. To each pair $(\xi, \eta) \in \mathfrak{g}_{1} \times \mathfrak{g}_{1}$, we assign the $m \times 2 n$ matrix $D(\xi, \eta):=$ $(\xi(1) \mid \eta(1))$. We will show that $\mathrm{rk} D(\xi, \eta) \leqslant n$ for any $(\xi, \eta) \in \overline{G_{0}(\mathfrak{c} \times \mathfrak{c})}$, whereas there are some $(\xi, \eta) \in \mathfrak{g}(1) \times \mathfrak{g}(1)$ such that $\operatorname{rk} D(\xi, \eta)>n$. This means that condition $(\mathbf{A})$ cannot be satisfied here.

Let $\mathfrak{c}(-1)$ be the set of $n \times m$ matrices

$$
\mathfrak{c}(-1)=\left\{\left(\begin{array}{ccccccc}
x_{1} & 0 & \ldots & 0 & 0 & \ldots & 0 \\
0 & x_{2} & \ldots & 0 & \vdots & \ldots & \vdots \\
\vdots & \vdots & \ddots & \vdots & \vdots & \ldots & \vdots \\
0 & \ldots & 0 & x_{n} & 0 & \ldots & 0
\end{array}\right) \mid x_{1}, \ldots, x_{n} \in \mathbb{k}\right\} .
$$

Then $\mathfrak{c}:=\left\{\left(\begin{array}{cc}0 & M \\ M^{t} & 0\end{array}\right) \mid M \in \mathfrak{c}(-1)\right\}$ is a Cartan subspace of $\mathfrak{g}_{1}$. It is clear from the definition that $\operatorname{rk} D(\xi, \eta) \leqslant n$ for any $(\xi, \eta) \in \mathfrak{c} \times \mathfrak{c}$. Next, an easy verification shows that this remains true for the elements of $G_{0}(\mathfrak{c} \times \mathfrak{c})$. Indeed, let $g=\left(\begin{array}{cc}B & 0 \\ 0 & C\end{array}\right) \in G_{0}$. Then $\operatorname{Ad}(g) \cdot \xi=\left(\begin{array}{cc}0 & B \xi(-1) C^{-1} \\ C \xi(1) B^{-1} & 0\end{array}\right)$. It follows that $D(\operatorname{Ad}(g) \cdot \xi, \operatorname{Ad}(g) \cdot \eta)=C D(\xi, \eta) \hat{B}$, where $\hat{B}=\left(\begin{array}{cc}B^{-1} & 0 \\ 0 & B^{-1}\end{array}\right)$. Hence $\operatorname{rk} D(\xi, \eta)=\operatorname{rk} D(\operatorname{Ad}(g) \cdot \xi, \operatorname{Ad}(g) \cdot \eta)$ for any $g \in G_{0}$.

On the other hand, it is clear that there are two $m \times n$ matrices $\xi(1), \eta(1)$, i.e., two elements of $\mathfrak{g}(1)$, such that $\operatorname{rk}(\xi(1) \mid \eta(1))=\min (2 n, m)>n$, since $m>n$. This completes our argument.

Thus, $\mathfrak{C}\left(\mathfrak{g}_{1}\right)$ has at least three irreducible components, by virtue of Corollary 4.2. However, this lower bound is far from being precise. Surprisingly, $\mathfrak{C}\left(\mathfrak{g}_{1}\right)$ can have arbitrary many irreducible components.

Proposition 4.4. Suppose $\left(\mathfrak{g}, \mathfrak{g}_{0}\right)=\left(\mathfrak{g l}_{n+m}, \mathfrak{g l}_{n} \oplus \mathfrak{g l}_{m}\right)$ with $n \leqslant m$. Then $\mathfrak{C}\left(\mathfrak{g}_{1}\right)$ has at least $2 \min (2 n, m)-2 n+1$ irreducible components.

Proof. For $\xi=\left(\begin{array}{cc}0 & Y \\ X & 0\end{array}\right) \in \mathfrak{g}_{1}$ and $\eta=\left(\begin{array}{cc}0 & U \\ Z & 0\end{array}\right) \in \mathfrak{g}_{1}$, we set $D_{1}(\xi, \eta):=(X \mid Z)$ (as above) and $D_{2}(\xi, \eta):=\left(Y^{t} \mid U^{t}\right)$. These are two $m \times 2 n$-matrices.

1. First, we are going to prove that $\operatorname{rk} D_{1}(\xi, \eta)+\operatorname{rk} D_{2}(\xi, \eta) \leqslant 2 n$ if $[\xi, \eta]=0$. Suppose rk $X=r$. Replacing $(\xi, \eta)$ with $(\operatorname{Ad}(g) \cdot \xi, \operatorname{Ad}(g) \cdot \eta)$ for a suitable $g \in G_{0}$, we may assume that $X=\left(x_{i j}\right)$ such that $x_{i i}=1$ if $i=1, \ldots, r$ and $x_{i j}=0$ for all other $i, j$. Then rk $D_{1}(\xi, \eta)=r+\mathrm{rk} Z_{1}$, where $Z_{1}$ is the $(m-r) \times n$ matrix consisting of the last $(m-r)$ rows of $Z$. Condition $[\xi, \eta]=0$ means that $X U=Z Y$ and $Y Z=U X$. The last $r$ rows of $X$ and hence of $X U$ are zero. Therefore $Z_{1} Y=0$ and $\operatorname{rk} Y \leqslant n-\operatorname{rk} Z_{1}$. 
Let $X_{0}, Z_{0}, U_{0}$ be the sub-matrices of $X, Z$, and $U$, respectively, consisting of the first $r$ rows. From the equality $X U=Z Y$ and our assumption on $X$, we then obtain $Z_{0} Y=$ $X_{0} U=U_{0}$. Therefore the rank of the $m \times(n+r)$ matrix $\left(Y^{t} \mid U_{0}^{t}\right)$ is not grater than $\operatorname{rk} Y$ and hence $\operatorname{rk}\left(Y^{t} \mid U^{t}\right) \leqslant \operatorname{rk} Y+(n-r)$. Thus,

$$
\operatorname{rk} D_{1}(\xi, \eta)+\operatorname{rk} D_{2}(\xi, \eta) \leqslant\left(r+\operatorname{rk} Z_{1}\right)+\left(n-\operatorname{rk} Z_{1}+n-r\right)=2 n .
$$

2. Let $P_{q}$ be the closed subset of $\mathfrak{C}\left(\mathfrak{g}_{1}\right)$ defined by the conditions:

$$
\operatorname{rk} D_{1}(\xi, \eta) \leqslant q \text { and } \operatorname{rk} D_{2}(\xi, \eta) \leqslant 2 n-q .
$$

As was just proved, $\mathfrak{C}\left(\mathfrak{g}_{1}\right)=\bigcup P_{q}$. Since $D_{1}(\xi, \eta)$ is an $m \times 2 n$-matrix, $\operatorname{rk} D_{1}(\xi, \eta) \leqslant$ $\min (2 n, m)$. Hence $P_{q} \subset P_{\min (2 n, m)}$ if $q>\min (2 n, m)$. Similarly, for $q<2 n-\min (2 n, m)$, we have $P_{q} \subset P_{r}$, where $r=2 n-\min (2 n, m)$. This implies that

$$
\mathfrak{C}\left(\mathfrak{g}_{1}\right)=\bigcup_{2 n-\min (2 n, m)}^{\min (2 n, m)} P_{q} .
$$

Let us show that $P_{q} \not \subset \bigcup_{r \neq q} P_{r}$ for each $q \in[2 n-\min (2 n, m), \min (2 n, m)]$. Since $\mathfrak{C}\left(\mathfrak{g}_{1}\right)$ is invariant under the transpose, there is no harm in assuming that $q \geqslant n$.

Keep the previous notation. Take $(\xi, \eta) \in \mathfrak{C}\left(\mathfrak{g}_{1}\right)$ such that the submatrices $X_{0}, Z_{0}, U_{0}$, etc. satisfy the following conditions: $\operatorname{rk} X=\operatorname{rk} X_{0}=n$ (i.e., $r=n$ ), $U=0, \operatorname{rk} Z_{1}=q-n$, and $Z_{0}=0$. Let $Y_{0}$ and $Y_{1}$ denote the submatrices of $Y$ consisting of the first $n$ columns and the last $m-n$ columns, respectively. Then the condition $[\xi, \eta]=0$ is equivalent to that $Z_{1} Y=0$ and $Y_{1} Z_{1}=0$. One can choose $Y$ satisfying these conditions and such that $Y_{1}=0$ and $\operatorname{rk} Y_{0}=n-\operatorname{rk} Z_{1}$. Then $\operatorname{rk} D_{1}(\xi, \eta)=q$ and $\operatorname{rk} D_{2}(\xi, \eta)=2 n-q$. That is, we found a point in $P_{q} \backslash \bigcup_{r \neq q} P_{r}$. It follows that each $P_{q}$ contains an irreducible component of $\mathfrak{C}\left(\mathfrak{g}_{1}\right)$ that does not belong to the other $P_{r}$, and we are done.

Remark. Motivated by the formula of Proposition 4.4 we set $F(n, m)=2 \min (2 n, m)-$ $2 n+1$ if $1 \leqslant n \leqslant m$. For $n=1$ and $m \geqslant 2$, we have $\operatorname{rk}\left(\mathfrak{g l}_{m+1}, \mathfrak{g l}_{m} \oplus \mathfrak{g l}_{1}\right)=1$, and it was shown in [12] that $\mathfrak{C}\left(\mathfrak{g}_{1}\right)$ has three irreducible components. Since $F(1, m)=3$ if $m \geqslant 2$, Proposition 4.4 gives here the exact value for the number of irreducible components. For $m=n$, we also obtain the exact number of irreducible components, in view of Theorem 3.3. It is curious to observe that $F(n, m)=F(m-n, m)$. Hence $F(m-1, m)=3$, and one may ask whether it is true that, for the symmetric pair $\left(\mathfrak{g l}_{2 m-1}, \mathfrak{g l}_{m} \oplus \mathfrak{g l}_{m-1}\right)$, the variety $\mathfrak{C}\left(\mathfrak{g}_{1}\right)$ has exactly three irreducible components. More generally, one may conjecture that the varieties $\left\{P_{q}\right\}$ in the previous proof are irreducible and therefore the number of irreducible components is always equal to $F(n, m)$. The equality $F(n, m)=F(m-n, m)$ also suggests that there might be a natural bijection between the irreducible components of the commuting varieties associated with $\left(\mathfrak{g l}_{m+n}, \mathfrak{g l}_{m} \oplus \mathfrak{g l}_{n}\right)$ and $\left(\mathfrak{g l}_{2 m-n}, \mathfrak{g l}_{m} \oplus \mathfrak{g l}_{m-n}\right)$. 
Example 4.5. $\left(\mathfrak{g}, \mathfrak{g}_{0}\right)=\left(\mathfrak{s o}_{2 n}, \mathfrak{g l}_{n}\right)$.

Let $V$ be a $2 n$-dimensional vector space and $\mathfrak{g}=\mathfrak{s o}(V)=\mathfrak{s o}_{2 n}$. Consider a decomposition of $V$ into a direct sum of two isotropic subspaces $V=V_{+} \oplus V_{-}$. Let $A \in \mathrm{O}(V)$ be such that $\left.A\right|_{V_{+}}=\mathrm{id}$ and $\left.A\right|_{V_{-}}=-\mathrm{id}$. Then the conjugation by $A$ defines an involution of $\mathfrak{g}$ such that $\mathfrak{g}_{0}=\mathfrak{g l}_{n} \cong \mathfrak{g l}\left(V_{+}\right) \cong \mathfrak{g l}\left(V_{-}\right)$and $\mathfrak{g}_{1} \subset \operatorname{Hom}\left(V_{+}, V_{-}\right) \oplus \operatorname{Hom}\left(V_{-}, V_{+}\right)$. More precisely, one can choose bases for $V_{+}$and $V_{-}$such that

$$
G_{0}=\left\{\left(\begin{array}{cc}
B & 0 \\
0 & \left(B^{t}\right)^{-1}
\end{array}\right) \mid B \in \mathrm{GL}_{n}\right\}, \mathfrak{g}_{1}=\left\{\left(\begin{array}{cc}
0 & X \\
Y & 0
\end{array}\right) \mid X, Y \in \mathfrak{g l}_{n}, X=-X^{t}, Y=-Y^{t}\right\} .
$$

Here $\mathfrak{g}(1)=\mathfrak{g}_{1} \cap \operatorname{Hom}\left(V_{+}, V_{-}\right)$and $\mathfrak{g}(-1)=\mathfrak{g}_{1} \cap \operatorname{Hom}\left(V_{-}, V_{+}\right)$. For a pair $(\xi, \eta) \in \mathfrak{g}_{1} \times \mathfrak{g}_{1}$ with $\xi=\left(\begin{array}{cc}0 & X \\ Y & 0\end{array}\right)$ and $\eta=\left(\begin{array}{cc}0 & Z \\ U & 0\end{array}\right)$, we set $D_{1}(\xi, \eta):=(X \mid Z)$. We take the Cartan subspace $\mathfrak{c} \subset \mathfrak{g}_{1}$ consisting of skew-symmetric anti-diagonal matrices, i.e.,

$$
\mathfrak{c}=\left\{\left(\begin{array}{cc}
0 & X \\
X & 0
\end{array}\right) \mid X=-X^{t} \text { and } X=\left(x_{i j}\right), x_{i j}=0 \text { if } i+j \neq n+1\right\} .
$$

Now assume that $n=2 k+1$. Then $\operatorname{rk} D_{1}(t, h)<n$ for any $(t, h) \in \mathfrak{c} \times \mathfrak{c}$. If $g=\left(\begin{array}{cc}B & 0 \\ 0 & \left(B^{t}\right)^{-1}\end{array}\right) \in G_{0}$ and $\xi=\left(\begin{array}{cc}0 & X \\ Y & 0\end{array}\right) \in \mathfrak{g}_{1}$, then $\operatorname{Ad}(g) \cdot \xi=\left(\begin{array}{cc}0 & B X B^{t} \\ \left(B^{t}\right)^{-1} Y B^{-1} & 0\end{array}\right)$. Therefore, $D_{1}(\operatorname{Ad}(g) \cdot \xi, \operatorname{Ad}(g) \cdot \eta)=B D_{1}(\xi, \eta) \hat{B}$, where $\hat{B}=\left(\begin{array}{cc}B^{t} & 0 \\ 0 & B^{t}\end{array}\right)$ is a non-degenerate $2 n \times 2 n$ matrix. Hence $\operatorname{rk} D_{1}(\operatorname{Ad}(g) \cdot \xi, \operatorname{Ad}(g) \cdot \eta)=\operatorname{rk} D_{1}(\xi, \eta)$ and $\operatorname{rk} D_{1}(t, h)<n$ for each pair $(t, h) \in \mathfrak{C}_{0}$.

Let $X, Z \in \mathfrak{g}(1)$ be skew-symmetric $n \times n$ matrices of rank $2 k$ such that the last column and the last row of $X$ are zero, and the first row and the first column of $Z$ are zero. Clearly, if $k \geqslant 1$, then $\operatorname{rk}(X \mid Z)=2 k+1=n$ and therefore $(X, Y) \notin \overline{G_{0}(\mathfrak{c}(1) \times \mathfrak{c}(1))}$. Thus, for $\left(\mathfrak{s o}_{2 n}, \mathfrak{g l}_{n}\right)$ with odd $n \geqslant 3$, condition ( $)$ is not satisfied.

Example 4.6. Consider now the symmetric pair $\left(\mathcal{E}_{6}, \mathfrak{s o}_{10} \oplus \mathfrak{t}_{1}\right)$.

Here $\mathfrak{g}(1)$ and $\mathfrak{g}(-1)$ are different half-spinor representations of $\mathfrak{s o}_{10}$. Let $\mathfrak{t}$ be a Cartan subalgebra of $\mathfrak{s o}_{10}$ and $\left\{\varepsilon_{1}, \ldots, \varepsilon_{5}\right\}$ an orthonormal basis of $\mathfrak{t}_{\mathbb{Q}}^{*}$. Let $\left\{\pi_{1}, \ldots, \pi_{5}\right\}$ be the fundamental weights of $\mathfrak{s o}_{10}$ such that

$$
\pi_{4}=\left(\varepsilon_{1}+\varepsilon_{2}+\varepsilon_{3}+\varepsilon_{4}-\varepsilon_{5}\right) / 2 \text { and } \pi_{5}=\left(\varepsilon_{1}+\varepsilon_{2}+\varepsilon_{3}+\varepsilon_{4}+\varepsilon_{5}\right) / 2
$$

are the highest weights of half-spinor representations. (See [9, Reference Chapter] for more details on the notation.) Let $\mathcal{R}(\varphi)$ denote the simple $\mathfrak{s o}_{10}$-module with highest weight $\varphi$. We assume that $\mathfrak{g}(1)=\mathcal{R}\left(\pi_{5}\right)$ and $\mathfrak{g}(-1)=\mathcal{R}\left(\pi_{4}\right)$. The rank of $\left(\mathcal{E}_{6}, \mathfrak{s o}_{10} \oplus \mathfrak{t}_{1}\right)$ equals 2 and a Cartan subspace $\mathfrak{c} \subset \mathfrak{g}_{1}$ can be chosen such that $\mathfrak{c}(1)$ is a $\mathfrak{t}$-stable subspace of $\mathfrak{g}(1)$ with weights $\pi_{5}$ and $\left(\varepsilon_{1}-\varepsilon_{2}-\varepsilon_{3}-\varepsilon_{4}-\varepsilon_{5}\right) / 2$. 
Because the weights of $\mathfrak{c}(1)$ belong to an open halfspace of $\mathfrak{t}_{\mathbb{Q}}^{*} \mathfrak{c}(1) \times \mathfrak{c}(1)$ lies in the nullcone of the $\operatorname{Spin}_{10}$-module $\mathfrak{g}(1) \times \mathfrak{g}(1)$. (Actually, similar assertion holds for any number of copies of $\mathfrak{c}(1)$ and $\mathfrak{g}(1)$. Therefore if $f \in \mathbb{k}[\mathfrak{g}(1) \times \mathfrak{g}(1)]^{\mathrm{Spin}_{10}}$ is a homogeneous polynomial of positive degree, then it vanishes on $\overline{G_{0} \cdot(\mathfrak{c}(1) \times \mathfrak{c}(1))}$. Hence the very existence of non-trivial Spin $_{10}$-invariant polynomials on $\mathfrak{g}(1) \times \mathfrak{g}(1)$ will imply that $(\mathbf{P})$ is not satisfied.

As $\mathcal{S}^{2}\left(\mathcal{R}\left(\pi_{4}\right)\right)=\mathcal{R}\left(2 \pi_{4}\right) \oplus \mathcal{R}\left(\pi_{1}\right)$, there are the following $\operatorname{Spin}_{10}$-equivariant inclusions:

$$
\mathcal{S}^{4}\left(\mathcal{R}\left(\pi_{4}\right) \oplus \mathcal{R}\left(\pi_{4}\right)\right) \supset \mathcal{S}^{2}\left(\mathcal{R}\left(\pi_{4}\right)\right) \otimes \mathcal{S}^{2}\left(\mathcal{R}\left(\pi_{4}\right)\right) \supset \mathcal{R}\left(\pi_{1}\right) \otimes \mathcal{R}\left(\pi_{1}\right) .
$$

Since $\left(\mathcal{R}\left(\pi_{1}\right) \otimes \mathcal{R}\left(\pi_{1}\right)\right)^{\operatorname{Spin}_{10}} \neq 0$, we obtain a desired non-trivial $\operatorname{Spin}_{10}$-invariant of degree 4 in $\mathbb{k}[\mathfrak{g}(1) \times \mathfrak{g}(1)]=\mathcal{S}^{\bullet}\left(\mathcal{R}\left(\pi_{4}\right) \oplus \mathcal{R}\left(\pi_{4}\right)\right)$.

\section{PRINCIPAL NILPOTENT PAIRS AND COMMUTING VARIETIES}

In this section, we prove that $\mathfrak{C}\left(\mathfrak{g}_{1}\right)$ is reducible for two symmetric pairs related to exceptional Lie algebras.

Let $\mathfrak{s}$ be the centraliser in $\mathfrak{g}$ of a generic element of $\mathfrak{c}$. Since $\mathfrak{c}$ is diagonalisable, $\mathfrak{g}_{x} \cap \mathfrak{g}_{y} \supset \mathfrak{s}$ for any $x, y \in \mathfrak{c}$. Therefore, $\operatorname{dim}\left(\mathfrak{g}_{x} \cap \mathfrak{g}_{y}\right) \geqslant \operatorname{dim} \mathfrak{s}$ for any pair $(x, y) \in \overline{G_{0}(\mathfrak{c} \times \mathfrak{c})}=\mathfrak{C}_{0}$. In what follows, we write $\mathfrak{g}_{x, y}$ for $\mathfrak{g}_{x} \cap \mathfrak{g}_{y}$. Assume that we managed to find a pair of commuting elements $\xi, \eta$ in $\mathfrak{g}_{1}$ such that $\operatorname{dim} \mathfrak{g}_{\xi, \eta}<\operatorname{dim} \mathfrak{s}$. Then clearly $(\xi, \eta) \notin \mathfrak{C}_{0}$, and we conclude that $\mathfrak{C}\left(\mathfrak{g}_{1}\right)$ is reducible.

Recall that $\operatorname{dim} \mathfrak{g}_{\xi, \eta} \geqslant \mathrm{rk} \mathfrak{g}$ for any pair of commuting elements. This follows from the irreducibility of the "usual" commuting variety $\mathfrak{C}(\mathfrak{g})$. Therefore, one may try to find a symmetric pair such that $\operatorname{dim} \mathfrak{s}>\operatorname{rk} \mathfrak{g}$, whereas $\operatorname{dim} \mathfrak{g}_{\xi, \eta}=\operatorname{rk} \mathfrak{g}$ for some $(\xi, \eta) \in \mathfrak{C}\left(\mathfrak{g}_{1}\right)$. This will be done with the help of theory of principal nilpotent pairs.

Definition 2 (Ginzburg [4]). A pair $\left(e_{1}, e_{2}\right) \in \mathfrak{g} \times \mathfrak{g}$ is said to be principal nilpotent (pn-pair, for short) if

$$
\left[e_{1}, e_{2}\right]=0, \quad \operatorname{dim} \mathfrak{g}_{e_{1}, e_{2}}=\operatorname{rk} \mathfrak{g},
$$

and there exists a pair of semisimple elements $\left(h_{1}, h_{2}\right) \in \mathfrak{g} \times \mathfrak{g}$ such that ad $h_{1}$ and ad $h_{2}$ have integral eigenvalues and $\left[h_{1}, h_{2}\right]=0, \quad\left[h_{i}, e_{j}\right]=\delta_{i j} e_{j} \quad(i, j \in\{1,2\})$.

Since the eigenvalues of ad $h_{1}$ and ad $h_{2}$ are integral, we can consider the corresponding $\mathbb{Z} \times \mathbb{Z}$-grading of $\mathfrak{g}$ :

$$
\mathfrak{g}=\bigoplus \mathfrak{g}(i, j), \text { where } \mathfrak{g}(i, j)=\left\{x \in \mathfrak{g} \mid\left[h_{1}, x\right]=i x,\left[h_{2}, x\right]=j x\right\} .
$$

Notice that $e_{1} \in \mathfrak{g}(1,0)$ and $e_{2} \in \mathfrak{g}(0,1)$. Using this bi-grading, we define a symmetric pair $\left(\mathbb{Z}_{2}\right.$-grading) by letting $\mathfrak{g}_{0}=\bigoplus_{i+j \text { is even }} \mathfrak{g}(i, j)$. From this construction, it follows that $\left(e_{1}, e_{2}\right) \in \mathfrak{C}\left(\mathfrak{g}_{1}\right)$. If we are lucky and the resulting symmetric pair has the property that $\operatorname{dim} \mathfrak{s}>\operatorname{rk} \mathfrak{g}$, i.e., $\mathfrak{s}$ is not Abelian, then we certainly know that $\mathfrak{C}\left(\mathfrak{g}_{1}\right)$ is reducible. 
It was shown by Ginzburg [4] that the number of $G$-orbits of pn-pairs is finite, and a complete classification of such pairs in exceptional Lie algebras is obtained by Elashvili and Panyushev [4, Appendix]. Using that classification, it is not hard to find out which $p n$-pairs lead to symmetric pairs (as described above) such that $\operatorname{dim} \mathfrak{s}>\mathrm{rk} \mathfrak{g}$. Actually, there is a unique $p n$-pair with this property if $\mathfrak{g}$ is exceptional. This pair occurs in the next theorem.

Theorem 5.1. For the symmetric pairs $\left(\mathcal{E}_{7}, \mathfrak{s o}_{12} \oplus \mathfrak{s l}_{2}\right)$ and $\left(\mathcal{E}_{8}, \mathcal{E}_{7} \oplus \mathfrak{s l}_{2}\right)$, the variety $\mathfrak{C}\left(\mathfrak{g}_{1}\right)$ is reducible.

Proof. 1. For the simple Lie algebra $\varepsilon_{7}$, there is a pn-pair $\left(e_{1}, e_{2}\right)$ such that both $e_{1}, e_{2}$ belong to the $E_{7}$-orbit of type $\mathcal{A}_{4}+\mathcal{A}_{1}$. (See below an explicit construction of such a pair.) The bigrading corresponding to this pair is depicted in [11, Fig. 2]. This shows that $\operatorname{dim} \mathfrak{g}_{0}=69$ for the corresponding $\mathbb{Z}_{2}$-grading. Hence $\mathfrak{g}_{0} \simeq \mathfrak{s o}_{12} \oplus \mathfrak{\mathfrak { s l } _ { 2 }}$. For this symmetric pair, we have $[\mathfrak{s}, \mathfrak{s}] \simeq 3 \mathcal{A}_{1}$. Hence $\left(e_{1}, e_{2}\right) \notin \mathfrak{C}_{0}$.

2. The same $p n$-pair can be used for proving reducibility in the $\mathcal{E}_{8}$-case. Using the standard Lie algebra embedding $\mathfrak{g}:=\mathcal{E}_{7} \subset \mathcal{E}_{8}=: \tilde{\mathfrak{g}}$, the first symmetric pair can be regarded as a subpair of the second. This simply means that we have two embeddings $\mathfrak{g}_{0}=\mathfrak{s o}_{12} \oplus \mathfrak{s l}_{2} \subset \mathcal{E}_{7} \oplus \mathfrak{s} \mathfrak{l}_{2}=\tilde{\mathfrak{g}}_{0}$ and $\mathfrak{g}_{1} \subset \tilde{\mathfrak{g}}_{1}$. Then $\left(e_{1}, e_{2}\right) \in \mathfrak{C}\left(\mathfrak{g}_{1}\right) \subset \mathfrak{C}\left(\tilde{\mathfrak{g}}_{1}\right)$. As above, we wish to prove that $\left(e_{1}, e_{2}\right)$ does not belong to the irreducible component $\tilde{\mathfrak{C}}_{0}=\overline{\tilde{G}_{0}(\tilde{\mathfrak{c}} \times \tilde{\mathfrak{c}})}$. The two symmetric pairs in question have the same rank (namely, 4), so we actually may assume that $\tilde{\mathfrak{c}}=\mathfrak{c}$. For a generic element of $\mathfrak{c}$, its stabiliser in $\tilde{\mathfrak{g}}$ has the semisimple part of type $\mathcal{D}_{4}$. Therefore $\operatorname{dim} \tilde{\mathfrak{s}}=28+4=32$. On the other hand, we will prove that $\operatorname{dim} \tilde{\mathfrak{g}}_{e_{1}, e_{2}}=26<32$, which establishes the desired reducibility.

To this end, we need an explicit construction of the pn-pair $\left(e_{1}, e_{2}\right)$ in $\mathcal{E}_{7}$. According to classical results of E.B. Dynkin [3], $\varepsilon_{7}$ contains a unique (up to conjugation) maximal semisimple subalgebra of type $\mathcal{A}_{2}$. The embedding $\mathcal{A}_{2} \subset \mathcal{E}_{7}$ has many remarkable properties. For instance, $E_{7} / A_{2}$ is an isotropy irreducible homogeneous space. Here $\mathcal{E}_{7} / \mathcal{A}_{2}$ is the simple $\mathcal{A}_{2}$-module $\mathcal{R}\left(4 \pi_{1}+4 \pi_{2}\right)$. Let $\alpha, \beta, \alpha+\beta$ be the positive roots of $\mathcal{A}_{2}$. There are two non-equivalent choices of nontrivial pn-pairs in $\mathcal{A}_{2}$. One takes $\left(e_{1}, e_{2}\right)=\left(e_{\alpha}, e_{\alpha+\beta}\right)$ or $\left(e_{\beta}, e_{\alpha+\beta}\right)$. Then one easily verifies that these two pairs remain principal also in $\varepsilon_{7}$. Furthermore, one can prove that these two pairs are conjugate with respect to the group $E_{7}$, so one obtains a single conjugacy class of $p n$-pairs in $\mathcal{E}_{7}$.

In order to compute $\operatorname{dim} \tilde{\mathfrak{g}}_{e_{1}, e_{2}}$, we first decompose $\tilde{\mathfrak{g}}=\mathcal{E}_{8}$ as $\mathcal{E}_{7}$-module, and then further as $\mathcal{A}_{2}$-module. First we notice that

$$
\left.\tilde{\mathfrak{g}}\right|_{\mathcal{E}_{7}}=\mathfrak{g} \oplus 2 \mathcal{V} \oplus 3 \mathbb{I},
$$

where $\mathcal{V}$ is the 56-dimensional simple $\mathcal{E}_{7}$-module and $\mathbb{I}$ is the trivial one-dimensional module. Since $\left(e_{1}, e_{2}\right)$ is a $p n$-pair in $\varepsilon_{7}$, we have $\operatorname{dim} \mathfrak{g}_{e_{1}, e_{2}}=7$. To compute the fixed-point 
subspace of $\left(e_{1}, e_{2}\right)$ in $\mathcal{V}$, we use fact that $\left.\mathcal{V}\right|_{\mathcal{A}_{2}}=\mathcal{R}\left(6 \pi_{1}\right) \oplus \mathcal{R}\left(6 \pi_{2}\right)$, see [3, Table 24]. Finally, one easily computes using the above two presentations of $\left(e_{1}, e_{2}\right)$ that $\operatorname{dim} \mathcal{R}\left(6 \pi_{1}\right)^{e_{1}, e_{2}}=1$ and $\operatorname{dim} \mathcal{R}\left(6 \pi_{2}\right)^{e_{1}, e_{2}}=7$ (or vice versa). Altogether, we obtain

$$
\operatorname{dim} \tilde{\mathfrak{g}}_{e_{1}, e_{2}}=7+2(7+1)+3=26 .
$$

This completes the proof of reducibility in the $\varepsilon_{8}$-case.

\section{A FEW REMARKS ON THE REMAINING SYMMETRIC PAIRS}

There are three cases for which the irreducibility of $\mathfrak{C}\left(\mathfrak{g}_{1}\right)$ is not known yet. They are listed below, together with their Satake diagrams.

1) $\left(\mathfrak{s p}_{2 n+2 m}, \mathfrak{s p}_{2 n} \oplus \mathfrak{s p}_{2 m}\right), n \geqslant m \geqslant 2$

2)

$\left(\mathfrak{s o}_{4 n}, \mathfrak{g l}_{2 n}\right), n \geqslant 2$

3)

$\left(\mathcal{E}_{7}, \mathcal{E}_{6} \oplus \mathfrak{t}_{1}\right)$
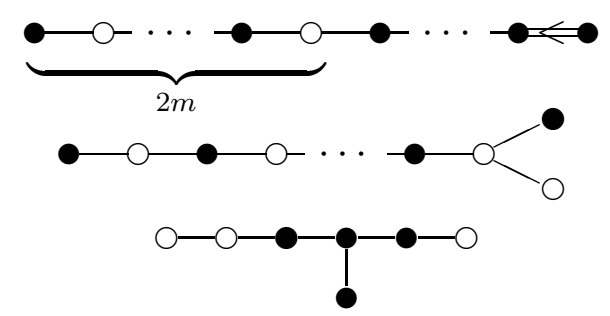

Items 2) and 3) are symmetric pairs of Hermitian type, i.e., $\mathfrak{g}_{0}$ is not semisimple. Such symmetric pairs (and the corresponding symmetric spaces) can be either of tube or nontube type. All symmetric pairs of Hermitian type arise form short $\mathbb{Z}$-gradings of $\mathfrak{g}$. This construction is explained in Section 4. There are many characterisations (definitions) of symmetric pairs of tube type. One of the possible definitions is the following. Let $G_{0}^{\prime}$ be the commutator group of $G_{0}$. Then $\left(\mathfrak{g}, \mathfrak{g}_{0}\right)$ is said to be of tube type if $\mathbb{k}[\mathfrak{g}(1)]^{G_{0}^{\prime}} \neq \mathbb{k}$. Then one can prove that $\left(\mathfrak{g}, \mathfrak{g}_{0}\right)$ is of tube type if and only if the dense (nilpotent) orbit in $G \cdot \mathfrak{g}(1)$ is even if and only if the $G_{0}^{\prime}$-action on $\mathfrak{g}(1)$ is stable. Yet another characterisation is that the symmetric pairs of tube type are those related to simple Jordan algebras.

Examples 4.3 (with $n \neq m$ ), 4.5 (with $n$ odd), and 4.6 represent all Hermitian symmetric pairs of non-tube type, and we have proved that for all these cases $\mathfrak{C}\left(\mathfrak{g}_{1}\right)$ is reducible. On the other hand, for the symmetric pair $\left(\mathfrak{s l}_{2 n}, \mathfrak{s l}_{n} \oplus \mathfrak{s l}_{n} \oplus \mathfrak{t}_{1}\right)$, which is of tube type, $\mathfrak{C}\left(\mathfrak{g}_{1}\right)$ is irreducible, as proved in Section 3, Other symmetric pairs of tube type are $\left(\mathfrak{s p}_{2 n}, \mathfrak{g l}_{n}\right)$ and $\left(\mathfrak{s o}_{n+2}, \mathfrak{s o}_{n} \oplus \mathfrak{s o}_{2}\right)$, where the irreducibility is also known. This suggests that for items 2$)$ and 3), which are of tube type, $\mathfrak{C}\left(\mathfrak{g}_{1}\right)$ ought to be irreducible, too. Hopefully, the connection with Jordan algebras might lead to a uniform proof of the irreducibility.

The first case is probably the most intricate. Here $\operatorname{rk}\left(\mathfrak{s p}_{2 n+2 m}, \mathfrak{s p}_{2 n} \oplus \mathfrak{s p}_{2 m}\right)=\min (n, m)=$ $m$. Since the commuting variety associated with $\left(\mathfrak{s p}_{2 n+2}, \mathfrak{s p}_{2 n} \oplus \mathfrak{s p}_{2}\right)$ is reducible, there is 
a non-zero semisimple element $h \in \mathfrak{g}_{1}$ such that $\mathfrak{C}\left(\mathfrak{g}_{1, h}\right)$ is reducible. Therefore, our induction scheme does not work in this situation. Nevertheless, using ad hoc methods the second author has proved that $\mathfrak{C}\left(\mathfrak{g}_{1}\right)$ is irreducible for $\left(\mathfrak{s p}_{2 n+4}, \mathfrak{s p}_{2 n} \oplus \mathfrak{s p}_{4}\right), n \geqslant 2$.

The problem of irreducibility of $\mathfrak{C}\left(\mathfrak{g}_{1, h}\right)$ also arises for second and third cases. The second case might be treated by induction, since all proper sub-symmetric pairs are direct sums of pairs of the same form and pairs $\left(\mathfrak{s l}_{2 m}, \mathfrak{s p}_{2 m}\right)$, where the irreducibility is known. For the third case, all proper sub-symmetric pairs $\left(\mathfrak{g}_{h}, \mathfrak{g}_{0, h}\right)$ can be found using Proposition 1.5. It turns out that here all commuting varieties $\mathfrak{C}\left(\mathfrak{g}_{1, h}\right)$ are irreducible.

Another question is whether a nilpotent $G_{0}$-orbit in $\mathfrak{g}_{1}$ gives rise to an irreducible component of $\mathfrak{C}\left(\mathfrak{g}_{1}\right)$ different from $\mathfrak{C}_{0}$. Provided all $\mathfrak{C}\left(\mathfrak{g}_{1, h}\right)$ are irreducible, this question can be reduced to non-even $\sigma$-distinguished nilpotent elements. A classification of $\sigma$ distinguished nilpotent orbits is deduced from Djoković's calculations [2]. According to Table 13 of [2], for item 3), there are two non-even $\sigma$-distinguished $G_{0}$-orbits in $\mathfrak{g}_{1}$. Thus, the task of verifying irreducibility of $\mathfrak{C}\left(\mathfrak{g}_{1}\right)$ in the third case seems to be manageable.

\section{REFERENCES}

[1] D. CollingWOOD and W. McGovern, "Nilpotent orbits in semisimple Lie algebras", Mathematics Series, Van Nostrand Reinhold, 1993.

[2] D.Ž. DJOKOvić, Classification of nilpotent elements in simple exceptional real Lie algebras of inner type and description of their centralizers, J. Algebra 112 (1988), no. 2, 503-524.

[3] E.B. DYNKIN, Semisimple subalgebras of semisimple Lie algebras, Matem. Sbornik 30(1952), № 2, 349462 (Russian). English translation: Amer. Math. Soc. Transl. II Ser., 6 (1957), 111-244.

[4] V. GinZBURG, Principal nilpotent pairs in a semisimple Lie algebra, I (with an appendix by A.G. Elashvili and D. Panyushev), Invent. Math. 140(2000), 511-561.

[5] J.C. JAntZEN, "Nilpotent orbits in representation theory", in: Progr. in Math., 228, 1-211, Birkhäuser Boston, 2004.

[6] B. Kostant and S. Rallis, Orbits and representations associated with symmetric spaces, Amer. J. Math. 93 (1971), 753-809.

[7] T. LEVASSEUR and J.T. STAFFORD, Invariant differential operators on the tangent space of some symmetric spaces. Ann. Inst. Fourier, 49, no. 6 (1999), 1711-1741.

[8] T. ОнтA, Classification of admissible nilpotent orbits in the classical real Lie algebras, J. of Algebra 136 (1991), 290-333.

[9] A.L. ONISHCHIK and E.B. VINBERG, "Lie groups and algebraic groups". Springer-Verlag, Berlin, 1990.

[10] D. PANYUSHEV, The Jacobian modules of a representation of a Lie algebra and geometry of commuting varieties, Compositio Math. 94 (1994), 181-199.

[11] D. PAnYUSHEV, Nilpotent pairs in semisimple Lie algebras and their characteristics, Intern. Math. Res. Notices no. 1 (2000), 1-21.

[12] D. PANYUSHEV, On the irreducibility of commuting varieties associated with involutions of simple Lie algebras, Funct. Anal. Appl. 38 (2004), 38-44.

[13] R. RiCHARDSON, Commuting varieties of semisimple Lie algebras and algebraic groups, Compositio Math. 38 (1979), 311-327. 
[14] H. SABOURIN and R.W.T. YU, Sur l'irréductibilité de la variété commutante d'une paire symétrique réductive de rang 1, Bull. Sci. Math. 126 (2002), 143-150.

[15] H. SABOURIN and R.W.T. YU, On the irreducibility of the commuting variety of the symmetric pair $\left(\mathfrak{s o}_{p+2}, \mathfrak{s o}_{p} \times \mathfrak{s o}_{2}\right)$, J. Lie Theory 16 (2006), no. 1, 57-65.

(D. Panyushev) Independent University of Moscow, Bol'shoi Vlasevskit PeR. 11, 119002 Moscow, RUSSIA

E-mail address: panyush@mccme.ru

(O. Yakimova) MPI FÜR MATHEMATIK, VIVATSGASSE 7, 53111 BonN, GERMANY

E-mail address: yakimova@mpim-bonn.mpg.de 